The University of Maine

\title{
DigitalCommons@UMaine
}

Earth Science Faculty Scholarship

Earth Sciences

1995

\section{A Wavelet Analysis of Pliopleistocene Climate Indicators: A New View of Periodicity Evolution}

Edward W. Bolton

Kirk A. Maasch

University of Maine - Main, kirk.maasch@maine.edu

Jonathan M. Lilly

Follow this and additional works at: https://digitalcommons.library.umaine.edu/ers_facpub

Part of the Earth Sciences Commons

\section{Repository Citation}

Bolton, Edward W.; Maasch, Kirk A.; and Lilly, Jonathan M., "A Wavelet Analysis of Pliopleistocene Climate Indicators: A New View of Periodicity Evolution" (1995). Earth Science Faculty Scholarship. 7.

https://digitalcommons.library.umaine.edu/ers_facpub/7 


\title{
A wavelet analysis of Plio-Pleistocene climate indicators: A new view of periodicity evolution
}

\author{
Edward W. Bolton, ${ }^{1}$ Kirk A. Maasch, ${ }^{2}$ and Jonathan M. Lilly ${ }^{13}$
}

\begin{abstract}
Wavelet analysis offers an alternative to Fourier based time-series analysis, and is particularly useful when the amplitudes and periods of dominant cycles are time dependent. We analyze climatic records derived from oxygen isotopic ratios of marine sediment cores with modified Morlet wavelets. We use a normalization of the Morlet wavelets which allows direct correspondence with Fourier analysis. This provides a direct view of the oscillations at various frequencies, and illustrates the nature of the time-dependence of the dominant cycles.
\end{abstract}

\section{Introduction}

Variability corresponding to 41 and $\sim 20 \mathrm{kyr}$ (kiloyear) periods observed in paleoclimate proxy records exhibit a high correlation with the variations in the Earth's orbital parameters (obliquity and precession, respectively) over the last few million years. In the late Pleistocene, a near- $100 \mathrm{kyr}$ cycle dominates the paleoclimate record, and is apparently in phase with weak eccentricity forcing in this frequency band. The $\sim 100$ kyr cycle is virtually absent before the mid-Pleistocene at around $900 \mathrm{ka}$ (kiloyears before present). Prior to this time, during the late Pliocene / early Pleistocene, the dominant period in paleoclimate proxy records was near $41 \mathrm{kyr}$. The mid-Pleistocene transition to a dominant $\sim 100 \mathrm{kyr}$ cycle has been the focus of numerous studies, and is a central issue in efforts to build a theory for the ice ages consistent with all available observations [Saltzman and Maasch, 1990]. Several techniques have been applied to paleoclimate records, such as evolutive spectral analysis [Joyce et al., 1990; Birchfield and Ghil, 1993] and envelope inversion [Park and Maasch, 1993], in an effort to quantify the onset of the $\sim 100 \mathrm{kyr}$ cycle. These techniques, which are extensions of traditional Fourier analysis, capture some of the time dependence of modal amplitudes. Wavelet analysis offers an alternative approach, with some advantages. Although there is always a tradeoff between time and frequency resolution (analogous to the uncertainty principle), the wavelet basis functions are local, rather than global, so that the local amplitude and phase of each "harmonic" may be more accurately estimated. In addition, the

\footnotetext{
${ }^{1}$ Department of Geology and Geophysics, Yale University

${ }^{2}$ Institute for Quaternary Studies and Department of Geological Sciences, University of Maine

${ }^{3}$ Now at School of Oceanography, University of Washington
}

Copyright 1995 by the American Geophysical Union.

Paper number 95GL02799

0094-8534/95/95GL-02799\$03.00 time resolution improves with increasing frequency, a property not shared by the above mentioned alternatives. We also use a wavelet normalization for which "period" and amplitude have meanings consistent with Fourier methods.

In this paper, we apply continuous wavelet transforms to time series derived from oxygen isotopic $\delta^{18} O$ measurements of planktonic foraminifera from Plio-Pleistocene marine sediment cores at ODP site 677 [Shackleton and Hall, 1989; and Shackleton et al. 1990], and ODP site 806 [Berger et al., 1993]. In addition, we analyze the SPECMAP stacked $\delta^{18} O$ record [Imbrie et al., 1984] and the July insolation curve for $65^{\circ} \mathrm{N}$ [Berger and Loutre, 1991].

The wavelet analysis presented here provides another view of the time dependence of "modal amplitudes" and suggests transitional states of $\sim 75 \mathrm{kyr}$ periodicity extant $\sim 750 \mathrm{ka}$. Climatic response at periods apparently unforced by orbital variations suggests the importance of mechanisms internal to the climate system, such as atmospheric / oceanic, lithospheric, ice sheet and carbon cycle processes. These involve slow-response feedbacks and nonlinear mechanisms, and could account for the changing amplitudes of the long periodicities.

\section{Wavelet Analysis}

Wavelet analysis had its origin in signal and image processing. Monographs published recently [Daubechies, 1992; Ruskai, et al., 1992] and a recent review by Farge [1992] will allow general access to this relatively new and rapidly expanding field. Much of the recent work on wavelets exploits orthogonal bases, due to efficient procedures for data compression and inversion. Nonorthogonal bases (utilized in continuous wavelet transforms) provide better phase and time resolution at all frequencies, and are thus the logical choice for our analysis of the climate record. The continuous wavelet transform provides a somewhat redundant view of the data, in that typically more coefficients are computed than the number of data points in the time series. The images of transforms and of envelopes of orthogonal pairs allow remarkable data visualization.

The continuous wavelet transform is the integral of the product of an analysis function and a time series. The analysis function is localized (in frequency content and time), and retains shape upon translation and rescaling its characteristic width. This allows better time resolution for high frequencies than for low frequencies. The continuous wavelet transform can be written: $C\left(a, t^{*}\right)=\int_{-\infty}^{\infty} g(x) f(t) d t$, with $x=\left(t-t^{*}\right) / a$, where $a$ is a scale parameter related to the characteristic period, $t^{*}$ represents evaluation times of the transformation, $g(x)$ is the analysis function, and $f(t)$ is the time 


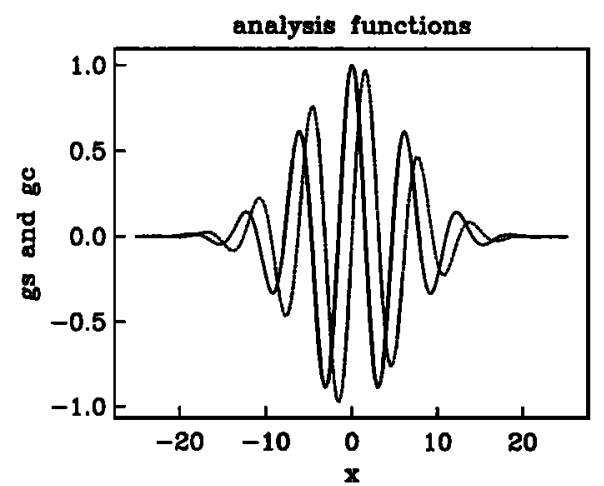

Figure 1. Morlet wavelets: The wavelets used in this analysis, in their non-normalized forms: $g_{s} / G_{s}$ (short dashed) and $g_{c} / G_{c}$ (solid line); both with $n=2$.

series under analysis. In practice, the integral limits may be replaced by values at which the wavelet amplitude is below some small threshold, and $t$ is evaluated at data points of the time series (which need not be the same times as $t^{*}$.

We consider the family of analysis functions which are sinusoids in a Gaussian envelope (modified Morlet wavelets). This family is well suited for adjusting the tradeoff between time and frequency resolution. We write $g_{s}(x, \zeta)=G_{s} \sin (x) W(\zeta x)$ and $g_{c}(x, \zeta)=$ $G_{c}(\cos (x)-\delta) W(\zeta x)$ where $W(\zeta x)=\exp \left(-\left((\zeta x)^{2}\right) / 2\right)$, with $x=\left(t-t^{*}\right) / a$, and $\zeta=1 /(n \pi)$, where $\delta$ is chosen to achieve zero mean. We have $\delta=\exp \left(-1 /\left(2 \zeta^{2}\right)\right)$, a factor which can be neglected for $n \gtrsim 2$ (as $\delta$ becomes small). For $n \gtrsim 1$ the parameter $n$ corresponds to the number of sinusoidal periods which fit between the inflection points of the Gaussian envelope. Although $n$ may be any real number, we restrict our analysis and normalizations to approximate forms valid for $n \gtrsim 1$. Larger values of $n$ (wider windows) allow better resolution in frequency, but worse resolution in time. The $n=2$ analysis functions are shown in Figure 1. Given a sinusoidal oscillation of angular wavenumber $\beta$, one can relate the scale parameter $a$ to the period $\tau=2 \pi / \beta$ by $a=\gamma_{m} \tau$ with $\gamma_{m}=1 /(2 \pi)$. This arises from the bandpass character of the $n \gtrsim 1$ Morlet wavelets, although for $n<1$ the $\tau: a$ scaling is more complicated. The real Morlet wavelet transforms of the data $f(t)$ may be defined as: $C_{c}\left(a, t^{*}\right)=\int_{-\infty}^{\infty} g_{c}(x) f(t) d t$, and $C_{s}\left(a, t^{*}\right)=\int_{-\infty}^{\infty} g_{s}(x) f(t) d t$. To examine the amplitude of an underlying sinusoidal signal, it is convenient to plot the modulus $E=\left(C_{s}^{2}+C_{c}^{2}\right)^{1 / 2}$ as a function of the period $(\tau)$ and time (Figure $2(A-D)$, with $n=2)$. At a particular time, the modulus may achieve a number of maxima (at various values of the scale parameter $a$ ). At such a local maxima, the signal under analysis locally contains significant amplitude at the corresponding period. Curved lines near the edges of Figure 2 (A-D) are limits of validity, placed at values of $t^{*}$ where the inflection point of the Gaussian envelope touches the limits of the data. The distance of this curve from $t^{*}=0$ is also a measure of "leakage" of the resolution in time. Higher frequencies are resolved more accurately in time than are low frequencies. The characteristic window width , $\delta t_{\text {char }}$, can be defined as the time duration be- tween the inflection points of the Gaussian envelope, for which we have $\delta t_{\text {char }}=2^{1 / 2} \tau n$ (where $\tau$ is the the period $\tau=a / \gamma_{m}$ and $n$ is defined above). Although on a log scale, the transform produces equal fractional bandwidths of synthetic sinusoids, the results indicate times at which bandwidths are broadened (eg. at rapid transitions).

Multitaper analysis (see Park and Maasch [1993]) can be performed in an evolutive sense with a moving window. Figure $2 \mathrm{E}$ shows the results of an evolutive multitaper spectral analysis, with $3-2 \pi$ prolate tapers $(N W=2, K=3$ in the notation of Thomson [1982], where $N W$ is the time/bandwidth product and $K$ is the number of tapers used), where the window width is 400 $\mathrm{kyr}$ and the time increment is $20 \mathrm{kyr}$. Both analyses indicate maximum amplitudes of the $41 \mathrm{kyr}$ cycle around $1500 \mathrm{ka}$. The wavelet and multitaper low frequency results are quite similar in their trends. The evolutive multitaper analysis lacks the improved time resolution at high frequencies provided by the wavelet analysis.

In order to recover amplitudes of the transforms $C_{c}$ and $C_{s}$ comparable to local modal amplitudes of periodic signals derived from Fourier methods, we choose a normalization such that the maximum of $C_{s}$ applied to $f(t)=\sin (\beta t)$ is unity and the maximum of $C_{c}$ applied to $f(t)=\cos (\beta t)$ is also unity, which occurs at $a=\gamma_{m} \tau=1 / \beta$ for $n \gtrsim 1$. This yields $G_{s}=1 /\left(a \rho_{s}\right)$ and $G_{c}=1 /\left(a \rho_{c}\right)$ with $\rho_{s}=\rho_{c}=\left((2 \pi)^{1 / 2}\right) /(2 \zeta)$ to leading order for $n \gtrsim 1$ (for $n<1$ different normalizations would be required for each of the two wavelets). The usual wavelet normalization (with $G \propto 1 / a^{1 / 2}$ ), recovers unit maximal magnitude upon the correlation of a wavelet with itself. Here we use a normalization (with $G \propto 1 / a$ - an $L^{1}$ norm) for which the wavelet transform of a unit amplitude sinusoid yields a maximal amplitude of unity, for sinusoids of any period. The $(1 / a)$ norm used here has been previously used (see for example Tchamitchian and Torrésani, 1992). It allows a direct comparison between wavelet and Fourier analyses.

No method of analysis is without undesirable artifacts. In the case of Fourier analysis, spectral leakage is well understood. The wavelets considered here have leakage of "frequency" between wavelet scales for which analytical expressions follow. Given a purely sinusoidal signal, of wavenumber $\beta$, one may calculate the "leakage" in wavelet parameter $a$, for the modified Morlet wavelets, with $\int_{-\infty}^{\infty} g_{c}\left(\frac{t}{a}\right) \cos (\beta t) d t=P[\exp (\varepsilon)+$ $\exp (-\varepsilon)-2] / \rho_{c}$ and $\int_{-\infty}^{\infty} g_{s}\left(\frac{t}{a}\right) \sin (\beta t) d t=P[\exp (\varepsilon)-$ $\exp (-\varepsilon)] / \rho_{s}$ where $P=(2 \pi)^{\frac{1}{2}} \exp \left[-\left((a \beta)^{2}+1\right) / 2 \zeta^{2}\right] / 2 \zeta$ and $\varepsilon=a \beta / \zeta^{2}$. If we write $\tau_{a}=2 \pi / \beta$ and look at the decay in amplitude of the transform in the vicinity of the maximum we have both the above integrals approximated by $(2 \pi)^{1 / 2} \exp \left[-\theta^{2} /\left(2 \zeta^{2}\right)\right] /\left(2 \zeta \rho_{c}\right)$ where $\theta=\delta \tau_{o} / \tau_{o}, \delta \tau_{o}=\tau-\tau_{o}$, and $\tau=a / \gamma_{m}$. Thus the fall off in the envelope amplitude would be symmetric if $\tau$ were expressed on a linear scale, and the distance between the inflection points of the Gaussian would be $\delta \tau_{\text {char }}=2 \tau_{o} /(n \pi)$. At a given time, the frequencies at which the envelope achieves local maximum amplitudes may be viewed as the important local carrier frequencies. These maxima typically form "ridges" through time of slowly changing frequencies, and it is along these ridges that the amplitudes of underlying oscillations are 


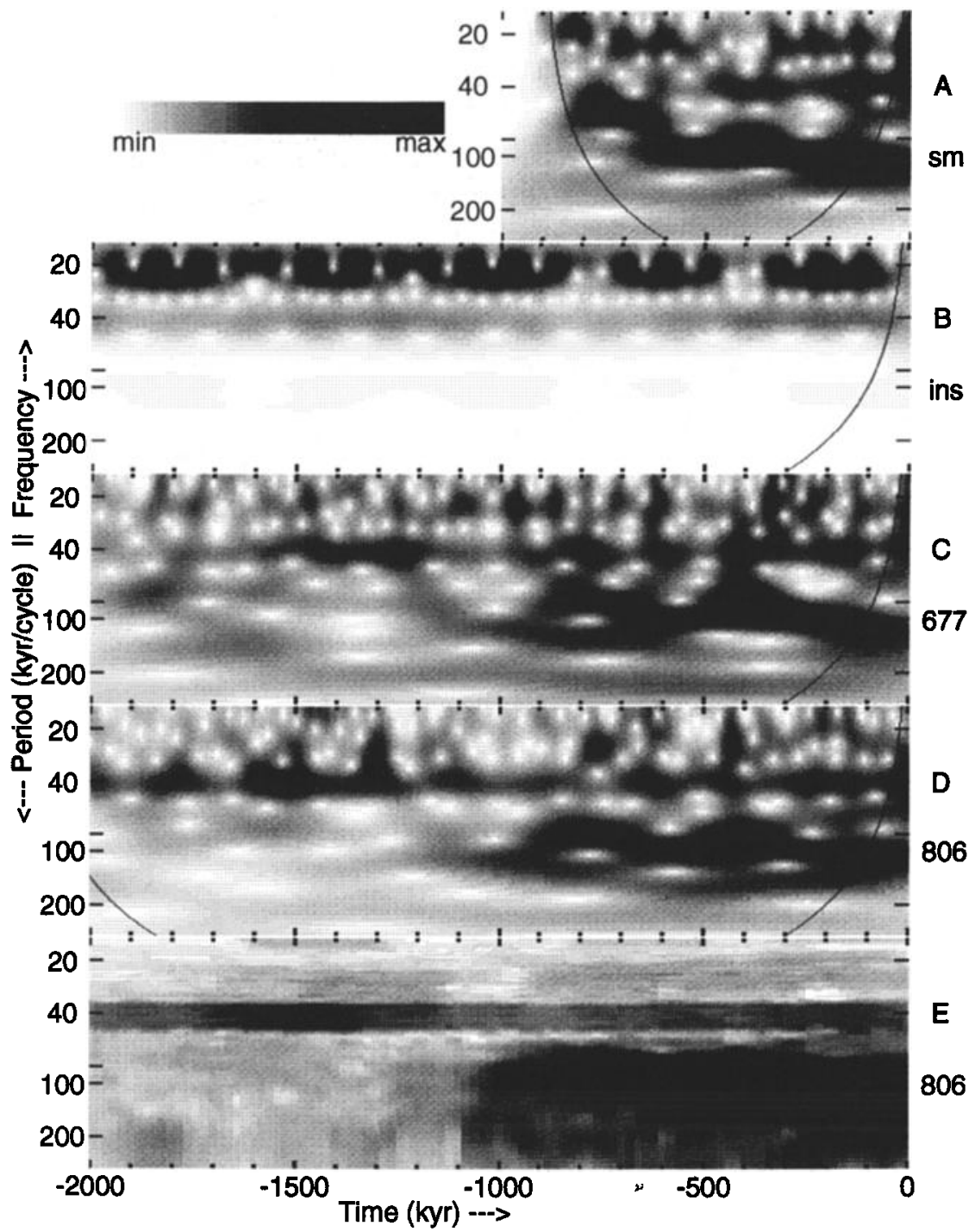

Figure 2. Wavelet and multitaper moduli of climate and insolation records: The first four parts correspond to wavelet analyses of (A) SPECMAP, (B) Insolation, (C) ODP 677, (D) ODP 806B. Parts (A)-(D) are images of the modulus $E=\left(C_{s}^{2}+\right.$ $\left.C_{c}^{2}\right)^{1 / 2}$, arising from the modified Morlet wavelets with $n=$ 2. These are estimates of the amplitudes of underlying sinusoidal oscillations at various periods. Note that the time resolution improves with increasing frequency for the wavelet envelopes. Part (E) is the multitaper evolutive spectral analysis (see text) of ODP 806B. The abscissa is time ( $t^{*}$, in kiloyears). The ordinate is a logarithmic period scale, with periods $(\tau)$ increasing downward. $\left(\tau=a / \gamma_{m}\right.$ in parts A-D - see text). Tick marks and labels are placed at locations corresponding to periods $(\tau)$ of $20,40,80$ (unlabeled), 100 and $200 \mathrm{kyr}$. The gray scale indicates the rescaled value of the modulus, with original maxima and units (for variation) of each part as (A) 0.955 (units of standard deviation of $\left.\delta^{18} \mathrm{O}\right)$, (B) $42.1\left(W / m^{2}\right)$, (C) $0.535\left(\delta^{18} O\right)$, (D) $0.36\left(\delta^{18} O\right)$, (E) arbitrary units proportional to modal amplitude. Each scale had zero as the minima. most accurately represented. Side bands in time arise from abrupt changes in signal amplitude. Time is better resolved for smaller $n$ and $a$ for the Gaussian-enveloped sinusoids. Frequency resolution improves with increasing $n$. The method presented works best for nearly periodic data. Users should be cautious when applying this method to data dominated by aperiodic episodes.

\section{Results and Discussion}

Numerous factors can influence the depth variation of $\delta^{18} \mathrm{O}$ in sediment cores, including global ice volume, local temperature, evaporation, bioturbation, compaction, variations in sedimentation rates of contributing components, and vital effects. The translation from depth to a time scale introduces other uncertainties. All of the above factors can contribute to the differences observed between various cores.

Analyses of insolation and planktonic $\delta^{18} O$ records from ODP 677, ODP 806, and SPECMAP (Fig. 2) show that all frequencies have strongly time dependent amplitudes. In some of the records, the $\sim 20 \mathrm{kyr}$ periodicity exhibits a beating pattern (with a period of $\sim 100 \mathrm{kyr}$ ), similar to that of insolation at $65^{\circ} \mathrm{N}$ (where the beating is caused by an interaction of 19 and $23 \mathrm{kyr}$ periods). This is particularly clear in the SPECMAP record. Note that the beating of the $\sim 20 \mathrm{kyr}$ cycle is not associated with any particular power in the $\sim 100$ kyr period band.
A persistent, though variable, $41 \mathrm{kyr}$ cycle is present in the wavelet transforms of sediment cores (shown in Fig. 2:A,C,D). The $\sim 20 \mathrm{kyr}$ cycle is present in all three of the $\delta^{18} O$ records, but appears to be weaker in ODP 806 than in ODP 677 or SPECMAP. This may be because the timescale for 806 was developed by tuning only to obliquity cycles, whereas timescales developed for 677 and SPECMAP were based on both precession and obliquity. Another possible reason for less variability of $\delta^{18} O$ in the precessional band at ODP 806 may be due to a small amplitude response of high latitude ice sheets at this frequency. Surface temperature in the western Pacific is relatively stable through glacial/interglacial cycles, thus minimizing the effect of local temperature on the fractionation of $\delta^{18} O$ in foraminifera tests [Matthews and Poore, 1980]. If ice sheets at high latitudes do not fluctuate with a large amplitude on a $\sim 20 \mathrm{kyr}$ time scale, then a $\delta^{18} O$ record that predominantly reflects global ice mass (such as ODP 806) would not exhibit much variability in the precession band. The signal of global ice mass contained in the record from ODP 677 is significantly overprinted with a temperature signal, perhaps due to the fact that this core is in a region affected by strong (and variable) upwelling. The strength of upwelling may be modulated by insolation changes in the precession band, which means the $\delta^{18} O$ record from this site would contain a stronger $\sim 20 \mathrm{kyr}$ component than for ODP 806 . 
In the modulus plots (Fig. 2:A-D) major changes are obvious in the lower frequency domain during the midPleistocene, giving rise to the dominant $\sim 100 \mathrm{kyr}$ cycle of the last $\sim 900 \mathrm{kyr}$. All three $\delta^{18} O$ records display similar patterns for the onset of the long periodicities. Transitional states are strongest at around $750 \mathrm{ka}$ which have most of their power at a period of $75 \mathrm{kyr}$ for ODP 806 and 677. In the SPECMAP record, while the pattern takes the same form, the actual timing ( $\sim 743 \mathrm{ka})$ and initial period ( $\sim 54 \mathrm{kyr}$ ) of the mid-Pleistocene transitional stage are different from those of ODP 677 and 806. This may be attributed to time scales errors in SPECMAP [Park and Maasch, 1993; Shackleton et al., 1990]. The progression of power toward lower frequencies, noted in the analysis of each of the cores during this time frame, does not correspond to any known external forcing. This points to changes internal to the Earth's climate system [Maasch and Saltzman, 1990]. The fact that a transition to longer periodicities occurred is well known, although the wavelet technique shows in greater detail how this change occurred.

\section{Conclusion}

The variability of $\delta^{18} O$ at frequencies corresponding to obliquity and precession, and the onset of a dominant $\sim 100 \mathrm{kyr}$ cycle in the mid-Pleistocene are all wellknown features of the paleoclimate record. However, the application of wavelet analysis to these $\delta^{18} O$ records provides us with a new view of this variability which allows us to see an overall picture of the time evolution as a function of frequency. This wavelet "fingerprint" of climate variability is more sensitive to variations in the higher frequency components than conventional Fourier-based spectral analysis techniques, owing to the localized nature of the wavelet analysis functions. Patterns of change in time-frequency space can potentially be recognized, even if they are distorted by timing errors. This is illustrated by the comparison between the modified Morlet wavelet transform of the SPECMAP record and the two ODP records (Fig. 2). In the region of time-frequency space near $800-900 \mathrm{ka}$, a $\sim 75 \mathrm{kyr}$ cycle emerges in the ODP 677 and $806 \delta^{18} \mathrm{O}$ records. In the SPECMAP record the transition has a similar form, but, it appears slightly later, and initially with a shorter period, than in the ODP records. As discussed above, we know that this is most likely due to a distorted time scale in this part of the SPECMAP record. However, it is important to note that the overall pattern - a mid-Pleistocene onset followed by a drift toward longer periods into the late Pleistocene - is common to all three of the $\delta^{18} O$ records.

Acknowledgments. We acknowledge discussions with R. Coifman, K. Sreenivasan, D. Lathrop, M. Mann, J. Park, B. Saltzman, R. B. Smith, G. Veronis, and L. Zubair. Special thanks to $M$. Mann for providing the evolutive multitaper analysis on which Figure $2 \mathrm{E}$ is based. The contribution to this work by KAM received support from NSF grant RII8922105 and NOAA grant NA36GP0258. EWB was supported by NSF grant EAR-92-04644, DOE grant DE-FG0290ER14153, and a research grant from Schlumberger-Doll Research, Rigefield, CT. Computations were made possible by NSF grant EAR-9018442. Initial results of this work were presented in Bolton, et al. [1993].

\section{References}

Berger, W. H., T. Bickert, H. Schmidt, and G. Wefer, Quaternary oxygen isotope record of pelagic foraminifers: site 806, Ontong Java Plateau, in Proc. ODP, Sci. Results, 130, (Ocean Drilling Program, College Station, TX), Berger, W. H., et al. Eds., 381-395, 1993.

Berger, A., and M. F. Loutre, Insolation values for the climate of the last 10 million years, Quat. Sci. Rev., 10, 297-317, 1991.

Birchfield ,G. E. and M. Ghil, Climate evolution in the Pliocene and Pleistocene from marine-sediment records and simulations: internal variability versus orbital forcing, J. Geophys. Res., 98, 10385-10399, 1993.

Bolton, E. W., K.A. Maasch and J.M. Lilly, A Wavelet Analysis of Plio-Pleistocene Climate Indicators: A New View of Periodicity Evolution, EOS, Trans. Am. Geoph. Union, 74, 366, 1993.

Daubechies, I., Ten Lectures on Wavelets, CBMS_NSF Regional Conf. Series in Appl. Math., 61, SIAM, Philadelphia, 1992.

Farge, M., Wavelet transforms and their applications to turbulence, Ann. Rev. Fluid Mech., 24, 395-457, 1992.

Imbrie, J., J.D. Hays, D.G. Martinson, A. McIntyre, A.C. Mix, J.J. Morley, N.G. Pisias, W.L. Prell, and N.J. Shackleton, The orbital theory of Pleistocene climate: support from a revised chronology of the marine $\delta^{18} O$ record, in Milankovitch and Climate, Part 1, Berger, A., J. Imbrie, J. Hays, G. Kukla, and B. Saltzman, Eds., (D. Riedel, Hingham, Mass.), 269-306., 1984.

Joyce, E. J., L. R. C. Tjalsma, and J. M. Prutzman, High-resolution planktic stable isotope record and spectral analysis for the last 5.35 M.Y.: Ocean Drilling Program site 625 Northeast Gulf of Mexico, Paleoceanography, 5, 507-529, 1990 .

Maasch, K. A. and B. Saltzman, A low-order dynamical model of global climatic variability over the full Pleistocene, J. Geophys. Res., 95, 1955-1963, 1990.

Matthews, R. K., and R. Z. Poore, Tertiary $\delta^{18} O$ record and glacio-eustatic sea-level fluctuations, Geology, 8, 501-504, 1980.

Park, J. and K. A. Maasch, Plio-Pleistocene time evolution of the 100-kyr cycle in marine paleoclimate records, $J$. Geophys. Res., 98, 447-461, 1993.

Ruskai, M. B. et al., Eds. Wavelets and Their Applications, Jones and Bartlett Publishers, Boston, 1992.

Saltzman, B., and K. A. Maasch, A first-order global model of the late Cenozoic climatic change, Trans. Roy. Soc. Edinburgh, 81, 315-325, 1990.

Shackleton, N. J., A. Berger, and W. R. Peltier, An alternative astronomical calibration of the lower Pleistocene timescale based on ODP site 677, Trans. R. Soc. Edinburgh, 81, 251, 1990.

Shackleton, N. J., and M. A. Hall, Stable isotope history of the Pleistocene at ODP site 677, Proc. Ocean Drill. Program Sci. Results, (Ocean Drilling Program, College Station, TX ), Becker, K., et al. Eds. 111, 295-316, 1989.

Tchamitchian, P. and B. Torrésani, Ridge and skeleton extraction from the wavelet transform, in Ruskai, M. B. et al., Eds. Wavelets and Their Applications, Jones and Bartlett Publishers, Boston, 1992.

Thomson, D. J., Spectrum estimation and harmonic analysis, Proc. IEEE, 7O, 1055-1096, 1982.

E. W. Bolton, Department of Geology and Geophysics, Kline Geology Laboratory, Yale University, P.O. Box 208109, New Haven, CT 06520-8109. (e-mail: bolton@milne.geology.yale.edu)

K. A. Maasch, Institute for Quaternary Studies and Department of Geological Sciences, University of Maine, 5711 Boardman Hall, Orono, ME 04469-5711. (e-mail: kirk@iceage.umeqs.maine.edu)

J. M. Lilly, School of Oceanography, WB-10 University of Washington Seattle, WA 98195.

(received August 4, 1994; revised March 22, 1995; accepted June 1, 1995.) 